\title{
Acoustic phonon mode anomalies in lanthanum doped lead zirconate-titanate relaxor ferroelectric ceramics
}

\author{
Ghulam Shabbir ${ }^{1, a)}$ and Seiji Kojima ${ }^{2, b)}$ \\ ${ }^{1}$ Physics Division, PINSTECH, Nilore, Islamabad 44000, Pakistan \\ ${ }^{2}$ Ultra-Broadband Spectroscopy Laboratory, Institute of Materials Science, University of Tsukuba, \\ Ibaraki 305-8573, Japan
}

(Received 17 September 2008; accepted 21 December 2008; published online 10 February 2009)

\begin{abstract}
Lanthanum-doped lead zirconate titanate, PLZT- $x / 65 / 35(x=8.0,9.0$, and 9.4), relaxor ferroelectric ceramics were investigated by the high resolution Brillouin light scattering spectroscopy in the temperature range from $\sim 870$ down to $\sim 170 \mathrm{~K}$. Broad anomalies were observed in the acoustic phonon mode velocity and related elastic stiffness coefficient showing minimum between $\sim 320-\sim 240 \mathrm{~K}$. The deviation in the acoustic mode velocity from the high temperature linearity at the Burns temperature $\left(T_{\mathrm{B}} \sim 720 \mathrm{~K}\right)$ and presence of a broad central peak in the temperature range $200 \leq T \leq 540 \mathrm{~K}$ were attributed to the dynamics of polar nanoregions with randomly oriented local polarization appearing due to site and/or charge disorder at both $A$ - and $B$-sites of the $\mathrm{ABO}_{3}$ perovskite lattice. The presence of a new temperature point $T_{\mathrm{d}}(\sim 575 \mathrm{~K})$ was conclusively established in PLZT-x/65/35 relaxor ceramics. (C) 2009 American Institute of Physics.
\end{abstract}

[DOI: $10.1063 / 1.3075833$ ]

\section{INTRODUCTION}

Lanthanum-doped lead zirconate-titanate (PLZT) relaxor ferroelectric (RFE) ceramics ${ }^{1}$ of the general formula, $\mathrm{Pb}_{1-3 x / 2} \mathrm{La}_{x}\left(\mathrm{Zr}_{y} \mathrm{Ti}_{1-y}\right) \mathrm{O}_{3}$, with $\mathrm{Zr} / \mathrm{Ti}: 65 / 35$ and $7<x<14$, (PLZT- $x / 65 / 35)$ have been a subject of intensive investigations due to their good dielectric, piezoelectric, electro-optic, and photostrictive properties for state of the art device applications. ${ }^{2}$ Photostriction is very important phenomenon for electromagnetic, noiseless, and remotely operated actuators which do not require external power source. Ferroelectricity is frustrated in RFEs because of site and/or charge occupancy disorder showing no structural phase transition and resemble glassy materials by exhibiting glasslike properties, e.g., aging, memory, and rejuvenation. ${ }^{3-5}$ The aging phenomenon in RFE materials is present not only below the freezing temperature $\left(T_{\mathrm{f}}\right)$, but extends even above the dielectric maximum $\left(T_{\mathrm{m}} \sim 350 \mathrm{~K}\right.$ for PLZT $)$. The relaxor state has been attributed to the appearance of polar nanoregions (PNRs) below the so-called Burns temperature $T_{\mathrm{B}}(\sim 620 \mathrm{~K}$ for PMN), with randomly oriented local polarization caused by the site and charge disorder and off-centering role of lead. The site and/or charge disorder in RFE materials gives rise to local quenched random fields and hence a transition to the long-range ordered state is prevented. However; long rage order can be induced by the application of sufficiently high electric field, which indicates that there exists a certain critical state in these materials. Field induced thermodynamic studies by Kutnjak et al. ${ }^{6}$ on $\mathrm{Pb}\left(\mathrm{Mg}_{1 / 3} \mathrm{Nb}_{2 / 3}\right)-\mathrm{PbTiO}_{3}$ (PMN-PT) have revealed that such criticality indeed exists and is not limited to PMN-PT but also seems to be found in PLZT RFEs.

PLZT-10/65/35 and PLZT-7.6/72/28 ceramics showed

\footnotetext{
${ }^{a}$ Electronic mail: gshabbir@gmail.com.

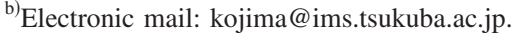

broad anomalies close to $T_{\mathrm{m}}$ (Ref. 7) in the acoustic properties measured by the Brillouin light scattering technique. It was suggested that the cooperative behavior of PNRs is more evident below $T_{\mathrm{B}}$ due to the development of shear deformation caused by the fluctuations in the local polarization, strain fields, and tilting of the oxygen octahedral. Central peak (CP) studies of the PMN-33\%PT crystal $^{8}$ supported these findings, and it was proposed that there exists a certain temperature point below $T_{\mathrm{B}}$ (in addition to $T_{\mathrm{B}}, T_{\mathrm{m}}$, and $T_{\mathrm{f}}$ ) where the dynamics of the PNRs produce sufficient local strain fields in the lattice resulting in appreciable dispersion in the response of acoustic phonon modes. Appearance of CPs in the light scattering spectra, giant electromechanical response, and peculiar nature of RFEs has brought them on the similar technological and phenomenological frontiers such as those of superconductors and colossal magnetoresistance materials. A pressure induced crossover from ferroelectric to relaxor state (in contrast to field induced relaxor to long-range ordered state) has been reported as a general feature of soft mode $\mathrm{ABO}_{3}$ perovskite ferroelectric materials. ${ }^{9}$ This was associated to the interactions and dynamics of PNRs originating from chemical substitution and lattice defects. Theoretical investigations of $\mathrm{PbTiO}_{3}$ at $0 \mathrm{~K}$ revealed a pressure induced transition to morphotropic phase boundary (MPB) (i.e., easy polarization rotation regime) suggesting that intrinsic disorder is not necessary for MPB and giant piezoelectric effect. ${ }^{10}$

This necessitates further detailed and more intriguing studies to be carried out on these materials to understand possible criticality and pressure-filed interplay. In perovskite ferroelectrics, structural phase transitions are induced by the fluctuations in the ionic displacements caused by the dipoledipole interactions triggered by the light scattering. Long wavelength acoustic phonons in solids are excited by the Brillouin light scattering in the frequency range $\sim 1.5$ to 


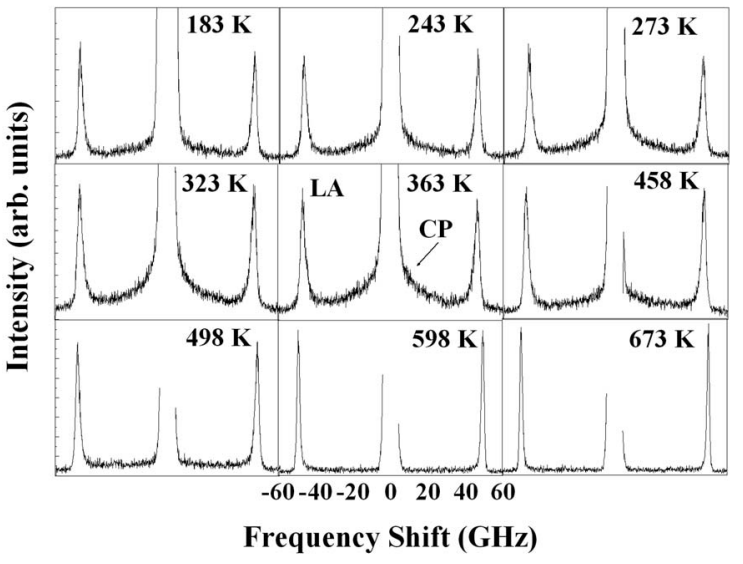

FIG. 1. Brillouin spectra of the PLZT-9.0/65/35 ceramic at some selected temperatures.

$\sim 60 \mathrm{GHz}$. Moreover, because phonons are created by the collective motions of atoms, which in turn directly reflect possible strain induced lattice instabilities. Therefore, Brillouin light scattering (a nondestructive technique) may be a very powerful tool to investigate the elastic properties of RFEs. In the present study we report our recent results on the high resolution micro-Brillouin light scattering study of the PLZT- $x / 65 / 35$ RFE ceramics.

\section{EXPERIMENTAL PROCEDURE}

Transparent PLZT- $x / 65 / 35$ ( $x=9.4,9.0$, and 8.0) ceramic specimens were prepared, by hotpressing in oxygen atmosphere. The samples were polished to optical quality, and high resolution micro-Brillouin scattering experiments were performed in the backscattering geometry by using a Sandercock $3+3$ pass tandem Fabry-Pérot interferometer in combination with an optical microscope (Olympus BH-2). For temperature dependent measurements a THMS600 heating stage (M/s Linkam, U.K.) was employed and acoustic phonon modes were excited by a diode-pumped solid-state laser (DPSS532) at a wavelength of $532 \mathrm{~nm}$ and a power of $\sim 100 \mathrm{~mW}$. All the measurements were made at a free spectral range (FSR) of $75 \mathrm{GHz}$. The temperature was first raised to $\geq 870 \mathrm{~K}$ and Brillouin spectra were then recorded in the cooling run with a temperature stability of $\pm 0.1 \mathrm{~K}$ and an accuracy of $\pm 1.0 \mathrm{~K}$.

\section{RESULTS AND DISCUSSIONS}

Typical Brillouin light scattering spectra of PLZT-9.0/ $65 / 35$ ceramic at some selected temperatures are plotted in Fig. 1. The measured spectra for all the specimens were consistent with results reported earlier ${ }^{7}$ and consist of a Raleigh peak and a Brillouin doublet arising from the longitudinal acoustic (LA) phonon mode. A strong $\mathrm{CP}$ was also observed in the temperature range between $T \approx 200-540 \mathrm{~K}$, depending on composition of sample. The Brillouin frequency shift $(\Delta \nu)$ and damping factor $(\Gamma)$, of the LA phonon modes were extracted from the observed spectra by describing the acoustic modes as damped harmonic oscillator function, $S_{\mathrm{DHO}}$, and

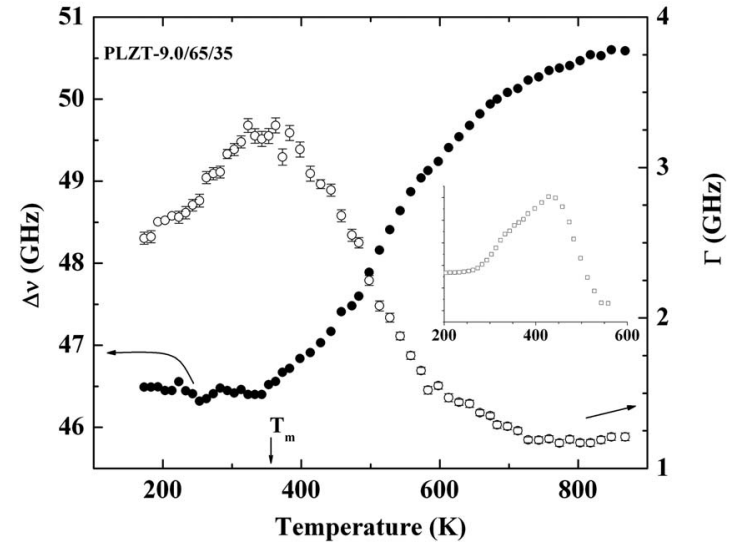

FIG. 2. Temperature dependences of the Brillouin frequency shift $(\Delta \nu)$ and damping factor $(\Gamma)$ for the PLZT-9.0/65/35 ceramic. Inset shows the observed CP intensity for the same specimen.

the CP (centered at zero frequency) by a Debye relaxation function, $S_{\mathrm{CP}}$, respectively. The fitting function thus can be decomposed as

$$
\begin{aligned}
& I(\nu) \infty\left\{S_{\mathrm{DHO}}+S_{\mathrm{CP}}+\mathrm{Ray}_{\mathrm{L}}\right\}+B_{\mathrm{g}}, \\
& S_{\mathrm{DHO}^{\infty}} \infty \sum_{i} \frac{F_{i} \Gamma_{i} \nu}{\left(\nu_{i}^{2}-\nu^{2}\right)^{2}+\Gamma_{i}^{2} \nu^{2}}, \\
& S_{\mathrm{CP}} \infty \frac{1}{1+\nu^{2} \tau^{2}},
\end{aligned}
$$

where Ray $_{\mathrm{L}}$ is Lorentzian function for Rayleigh peak, $B_{\mathrm{g}}$, is the background, and $F_{i}$ is related to the Bose-Einstein thermal factor, $n(\nu)=[\exp (h \nu / k T)]^{-1}$, where $h$ and $k$ stand for Plank constant and Boltzmann constant, respectively.

Temperature dependences of the Brillouin frequency shift and damping factor of the LA mode thus obtained are plotted in Fig. 2 for PLZT-9.0/65/35. The respective data for the other two samples exhibited similar trend and hence are not shown. As PLZT ceramics remain pseudocubic ( $a \sim 0.408 \mathrm{~nm}$ ) showing no structural phase transition in the investigated composition range, therefore the acoustic velocity, $V_{\mathrm{LA}}$, of the LA phonon mode corresponds to the elastic stiffness coefficient, $c_{11}\left(=c_{22}=c_{33}\right)$, and is related to $\Delta \nu$ by the following relation:

$$
\frac{2 \pi \Delta \nu}{q}=V_{\mathrm{LA}}=\sqrt{\frac{c_{11}}{\rho}},
$$

where $\rho=7863\left(\mathrm{~kg} \mathrm{~m}^{-3}\right)$ is density of the sample and $q$ $\left(\sim 0.062 \mathrm{~nm}^{-1}\right)$ is the scattering wave vector.

From Fig. 2 it can be seen that $\Delta \nu$ shows broad softening and a corresponding rise in phonon damping with decreasing temperature. The temperature dependences of the acoustic phonon mode velocities $\left(V_{\mathrm{LA}}\right)$ and the related elastic stiffness coefficients $\left(c_{11}\right)$ for the three PLZT compositions are plotted in Fig. 3. These data show that $V_{\mathrm{LA}}$ and $c_{11}$ both exhibit broad softening with decreasing temperature. The large values of elastic constants (present data and Ref. 7) depict the high load bearing capability of the actuating devices fabricated from PLZT $x / 65 / 35$ ceramics. It is also interesting to 


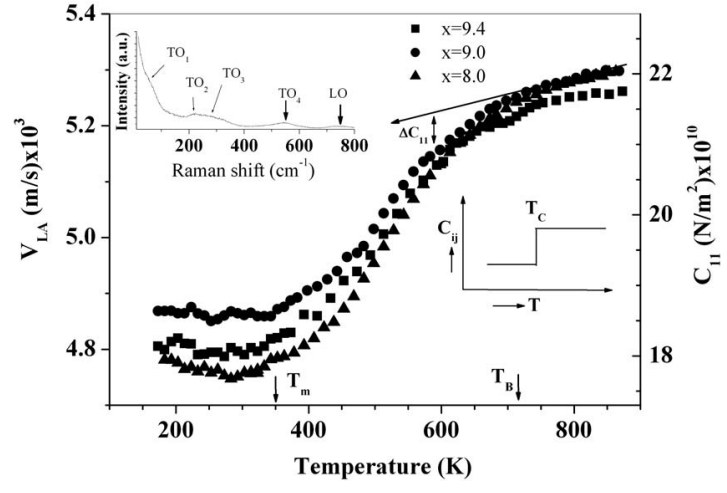

FIG. 3. Temperature dependences of the sound velocity $\left(V_{\mathrm{LA}}\right)$ and related elastic constant of the LA phonon modes for PLZT- $x / 65 / 35$ ceramics. The upper left inset shows room temperature Raman spectrum of PLZT-9.0/ $65 / 35$ lower right inset gives elastic constant versus temperature as predicted by Landau theory.

note that the observed temperature dependences of $V_{\mathrm{LA}}$ and $c_{11}$ are similar to that for PLZT-10/65/35 and PLZT-7.6/ $72 / 28$ RFE ceramics reported earlier. ${ }^{7}$ Room temperature Raman spectrum of PLZT-9.0/65/35 ceramic is also shown in Fig. 3 as an inset. Raman spectrum is forbidden in PLZT relaxor ceramics due to their average cubic symmetry. However, in PLZT-like disordered glassy ceramics broad bands are observed most probably connected with second order processes resulting from coupling of hard polar modes with the fluctuating local polarization.

To address the observed temperature dependences of the acoustic mode velocity and the elastic constant, one needs to understand the fundamental factors on which various microscopic models of relaxor physics are based. The first main factor accounted for relaxor phenomenon is the presence of inherent random site and/or charge disorder (ions are displaced from their crystallographic special positions of the ideal perovskite structure) that results in quenched random fields. These ionic displacements are expected to persist in RFEs both below and above $T_{\mathrm{B}}$. The second and most widely discussed one is the existence of random polar entities termed as PNRs which play central role in understanding the physical properties of RFEs. It has been widely accepted that relaxor properties are related to the compositionally induced site and charge disorder that has been considered as very origin of the PNRs (Ref. 11) but any exact connection between such disorder and PNRs still lacks enough quantitative evidence. Stock et al. ${ }^{12}$ have reported that PMN-60\%PT shows a long-range order transformation at $550 \mathrm{~K}$ such as pure $\mathrm{PbTiO}_{3}$, however, lattice dynamics are exactly same as that for prototypical RFEs PMN and PZN. This effect was associated to the random fields resulting from intrinsic chemical inhomogeneity. Nevertheless, it is thermal profile of these polar entities that defines the well known physical anomalies in RFEs (e.g., for PLZT- $x / 65 / 35$ see Fig. 4). Based on the dominant role of charge and lattice disorder, the spherical random-bond random-field model $^{13}$ was proposed to explain the disordered glasslike dynamics of RFEs. As to the random lattice disorder in RFEs, it arises from compositional inhomogeneities and ion off centering in the highly polarizable $\mathrm{ABO}_{3}$ host lattice. At very high temperatures

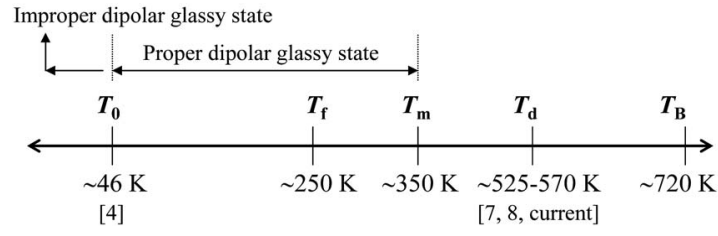

FIG. 4. Thermal profile of PLZT- $x / 65 / 35$ RFE ceramics, representing a series of transition anomalies.

$\left(T \gg T_{\mathrm{B}}\right)$, although the lattice disorder persists but the very existence of well defined dipole moments cannot be expected due to large random ionic fluctuations. With decreasing temperature, symmetry breaking occurs in RFEs at nanoscale resulting in the formation of PNRs (with randomly oriented, local polarization $P_{\mathrm{d}}$, such that $\Sigma P_{\mathrm{d}}=0$, however, $\Sigma\left(P_{\mathrm{d}}\right)^{2}$ $\neq 0)$ at $T_{\mathrm{B}}$, well above $T_{\mathrm{m}}$ what was observed by Burns and $\mathrm{Dacol}^{14}$ in the measurement of refractive index $n(T)$ versus temperature of PLZT-x/65/35 ceramics. The deviation in $n(T)$ of the high temperature linear part in normal ferroelectrics is expected to occur at $T_{\mathrm{c}}$ but a similar deviation in $n(T)$ was also observed in $\mathrm{BaTiO}_{3}$ not at $T_{\mathrm{c}}$ but rather between $T_{\mathrm{c}}$ and $\sim T_{\mathrm{c}}+180 \mathrm{~K} .{ }^{15}$ This peculiar observation, different to that expected from the soft mode theory, was associated to the local polarization fluctuations (no spontaneous polarization above $T_{\mathrm{c}}$ in $\mathrm{BaTiO}_{3}$ ) in analogy to other disordered materials caused by the quadratic electro-optic effect. Regarding ionic disorder the possible common factor to RFEs and $\mathrm{BaTiO}_{3}$ only may be the random shifts of Ti ions, very important source of ferroelectric phase transition. The acoustic properties of PMN-33\%PT RFE single crystal having a first order phase cubic-tetragonal structural transition at $T_{\mathrm{C}-\mathrm{T}}$ $\sim 415 \mathrm{~K}$ were attributed to the appearance and subsequent slowing down dynamics of PNRs. ${ }^{8}$ The growth in size of PNRs with temperature changes in PMN was evidenced by the high resolution transmission electron microscopy and it became $\sim 10 \mathrm{~nm}$ at $160 \mathrm{~K}$. Although any direct relation between PNRs and elastic properties observed in present study remains unclear but very broad acoustic anomalies (such as dielectric dispersion) are directly associated to the relaxation process of PNRs that in turn are probably linked to the local lattice dynamics in relaxor materials.

In the light of Landau theory for continuous phase transitions, when linear coupling with strain but quadratic in the order parameter is assumed, the change in elastic constant becomes $^{16}$

$$
\Delta c_{11}(T)=-g^{2}\left\langle P_{\mathrm{d}}^{2}\right\rangle \chi
$$

here, $g$ is the constant of electrostriction and $\chi$ is the susceptibility. For a structural phase transition, $c_{11}(T)$ (lower right inset in Fig. 3) should be unchanged in the paraelectric phase and for $T<T_{\mathrm{c}}$ the contributions from the order parameter and susceptibility cancel out therefore a steplike discontinuity should be observed in $c_{11}(T)$. The broad dispersion observed in $c_{11}(T)$ at $T_{\mathrm{B}}$ (Fig. 3), far away from $T_{\mathrm{c}}$ ( $T_{\mathrm{m}}$ in RFEs), is due to strong critical contribution of the order parameter and acoustic anomaly is therefore extended over a wide temperature range instead of sharp transition.

The local polarization in PLZT- $x / 65 / 35$ ceramics has been evidenced up to $640 \mathrm{~K} .{ }^{14}$ A qualitative look at Fig. 3 
depicts that the acoustic mode velocities of the three ceramics deviate from the high temperature linearity at $T_{\mathrm{B}} \sim 720 \mathrm{~K}$ and $\Delta V_{\mathrm{LA}} / \Delta T$ is enhanced below $T=T_{\mathrm{d}}$ $(\sim 575 \mathrm{~K})$ with decreasing temperature (from $\sim 6 \%$ between $T=728-573 \mathrm{~K}$ to $\sim 10 \%$ between $T \approx 573-408 \mathrm{~K})$. It is anticipated that $T_{\mathrm{d}}$ is the actual temperature below which cooperative characteristics of PNRs become more prominent due to development of shear deformation. ${ }^{7,8}$ This new intermediate temperature, $T_{\mathrm{d}}$, has also been reported by light scattering experiments for other relaxors such as PMN- $x \% \mathrm{PT},{ }^{8}$ PZN ${ }^{17,18}$ and PZN- $x \%$ PT (Ref. 19) single crystals. Therefore it seems appropriate to argue that $T_{\mathrm{B}}$ is an approximate temperature where the $P_{\mathrm{d}}$ is observed but probably PNRs make real sense at $T_{\mathrm{d}}$. Below $T_{\mathrm{B}}$, thermal fluctuations in the matrix are decreased to such an extent that the presence of $P_{\mathrm{d}}$ can be evidenced in the acoustic mode velocity. In the region $\sim 575 \leq T \leq 720 \mathrm{~K}$, the polarization fluctuations dominate due to growth of PNRs in size and density but the percolation limit cannot be reached. Hence a dynamic slowing down of PNRs occurs that impedes the true FE phase transition. At lower temperatures $200 \leq T \leq 540 \mathrm{~K}$, a strong CP was also observed (inset Fig. 2) exhibiting maximum at $T \sim 430 \mathrm{~K}$ and became very weak at $T \lesssim 250 \mathrm{~K}$. CP curve may be suitable only for qualitative analysis because accurate data need to be measured with a broader FSR. An increase in damping at $T<T_{\mathrm{B}}$, a maximum near $T_{\mathrm{m}}$, and a broad minimum in $c_{11}$ extending from $\sim 320$ to $\sim 240 \mathrm{~K}$ show that this region is predominated by fluctuations of the local polarization induced by the PNRs that ultimately freeze at $T_{\mathrm{f}}$ but still they can be put into order by applying a sufficiently high electric field. Nevertheless, complete freezing of polarization fluctuations of PNRs cannot be envisioned until $T_{0}$. The minor rise in $V_{\mathrm{LA}}$ or $c_{11}$ and decrease in damping below $T_{\mathrm{m}}$ indicates that certain degrees of freedom still prevail that are most probably frozen at $T_{0}$ where an elastic anomaly was observed with significant aging effect. ${ }^{4,5}$ Local cationic shifts of $\mathrm{Pb}^{2+}$ (a displacement of $\sim 0.5 \AA$ from the high symmetry position) due to asymmetric covalent bonds make it more isotropic than the strongly coordinated $B$-site ferroactive ions. The substitution of $\mathrm{La}^{3+}$ for $\mathrm{Pb}^{2+}$ at the $A$-site creates randomly distributed vacancies that give rise to an additional disorder. This additional disorder drives the system (PLZT- $x / 65 / 35)$ to a long-range ordered state for $x$ $<7$ at. $\%$ and relaxor state, for $7<x<14$ at. $\%$. It is therefore, anticipated that disorder due to $\mathrm{La}^{3+}$ and ion offcentering role of $\mathrm{Pb}^{2+}$ at $A$-site may be responsible for very low temperature relaxation dynamics of PLZT RFE ceramics. It is therefore proposed that series of transition anomalies observed in the thermal profile of PLZT relaxor ceramics originate from (as shown in Fig. 4): (a) paraelectric to relaxor transition at $T_{\mathrm{B}}$ due to formation of PNRs whose cooperative dynamics is more sensible below $T_{\mathrm{d}}$ and a strong dielectric dispersion at $T_{\mathrm{m}}$, (b) glasslike slowing down of relaxor dynamics below $T_{\mathrm{m}}$ results in freezing of PNRs at $T_{\mathrm{f}}$ while certain degrees of freedom related to $A$-site disorder remain active (and act as secondary order parameter) that probably freeze at $T_{0}$. This argument may be supported by the observation that a part of the relaxation spectrum remains unfrozen below $T_{\mathrm{f}}$ in PLZT-9.0/65/35 and PMN relaxors. ${ }^{20}$
The low temperature relaxor dynamics resembles to some dipolar glasses (Ref. 20 and references therein) at least qualitatively, and it is anticipated that anomaly at $T_{0}$ results from transition of "proper" to "improper dipolar glassy" state in analogy to improper ferroelectrics.

\section{SUMMARY AND CONCLUSION}

In conclusion, high resolution Brillouin light scattering technique was applied to study the elastic properties of PLZT- $x$ /65/35 RFE ceramics in a broad temperature range from $\sim 170$ to $\sim 870 \mathrm{~K}$. The observed Brillouin frequency shift and the acoustic phonon velocity and corresponding elastic stiffness coefficient for all the three ceramics exhibited a broad anomaly near $T_{\mathrm{m}}$ along with a corresponding maximum in the damping factor. The acoustic mode damping and velocities deviated from the high temperature linear behavior at $T_{\mathrm{B}} \sim 720 \mathrm{~K}$ that was associated with the gradual appearance of microscopic local polarization due to presence of charge/site disorder. It was observed that dynamics of PNRs are clearer below $T_{\mathrm{d}} \sim 575 \mathrm{~K}$ which seems to be a common feature of RFEs. The persistence of average cubic symmetry in RFEs down to very low temperatures indicates that certain degrees of freedom might be still active even below $T_{0}$ related to charge/site disorder at $A$-site and resulting PNRs. It is therefore anticipated that direct observation of any possible interaction between charge disorder and PNRs to very low temperatures is highly demanded that may provide necessary window for further experiments until a well defined physical model such as Landau theory is presented for these materials.

\section{ACKNOWLEDGMENTS}

Authors are grateful to Professor Q-R Yin of Shinghai Institute of Ceramics, the Chinese Academy of Sciences, for kindly providing good quality ceramic samples.

${ }^{1}$ G. H. Haertling and C. E. Land, J. Am. Ceram. Soc. 54, 1 (1971).

${ }^{2}$ G. H. Haertling, Ferroelectrics 75, 25 (1987).

${ }^{3}$ F. Cordero, F. Craciun, A. Franco, D. Piazza, and C. Galassi, Phys. Rev. Lett. 93, 097601 (2004).

${ }^{4}$ G. Shabbir, J.-H. Ko, and S. Kojima, Appl. Phys. Lett. 86, 012908 (2005).

${ }^{5}$ G. Shabbir and S. Kojima, J. Korean Phys. Soc. 46, 128 (2005).

${ }^{6}$ Z. Kutnjak, J. Petzelt, and R. Blinc, Nature (London) 441, 956 (2006).

${ }^{7}$ G. Shabbir and S. Kojima, J. Phys.: Condens. Matter 15, 7717 (2003).

${ }^{8}$ G. Shabbir and S. Kojima, Appl. Phys. Lett. 91, 062911 (2007).

${ }^{9}$ G. A. Samara and E. L. Venturini, Phase Transit. 79, 21 (2006).

${ }^{10}$ Z. Wu and R. E. Cohen, Phys. Rev. Lett. 95, 037601 (2005).

${ }^{11}$ V. Westphal, W. Kleemann, and M. D. Glinchuk, Phys. Rev. Lett. 68, 847 (1992).

${ }^{12}$ C. Stock, D. Ellis, I. P. Swainson, G. Xu, H. Hiraka, Z. Zhong, H. Luo, X. Zhao, D. Viehland, R. J. Birgeneau, and G. Shirane, Phys. Rev. B 73, 064107 (2006)

${ }^{13}$ R. Pirc and R. Blinc, Phys. Rev. B 60, 13470 (1999).

${ }^{14}$ G. Burns and F. H. Dacol, Phys. Rev. B 28, 2527 (1983).

${ }^{15}$ G. Burns and F. H. Dacol, Solid State Commun. 42, 9 (1982).

${ }^{16}$ W. Rehwald, Adv. Phys. 22, 721 (1973).

${ }^{17}$ O. Svitelskiy, D. La-Orauttapong, J. Toulouse, W. Chen, and Z.-G. Ye, Phys. Rev. B 72, 172106 (2005).

${ }^{18}$ J. Toulouse, F. Jiang, O. Svitelskiy, W. Chen, and Z.-G. Ye, Phys. Rev. B 72, 184106 (2005).

${ }^{19}$ J.-H. Ko, D. H. Kim, and S. Kojima, Phys. Rev. B 77, 104110 (2008).

${ }^{20}$ Z. Kutnjak, C. Filipic, R. Pirc, A. Levstik, R. Farhi, and M.-El. Marssi, Phys. Rev. B 59, 294 (1999). 